mutation, tau, in the hamster. Animals with one copy of the mutated gene show a 22-hour period in locomotor activity, and animals with two mutated copies have a 20 -hour periodicity $^{8}$. Unfortunately, further molecular analysis of the tau locus has not been easy, owing to the lack of well-developed genetic maps in hamsters.

The mouse, however, is genetically well characterized, and a number of genes have been identified that are rapidly induced by light in its $\mathrm{SCN}^{9}$. All of these genes encode transcription factors that contain structural motifs such as the leucine zipper, which mediates protein-protein interactions, and the zinc finger, which mediates protein-DNA interactions. Expression of some of these genes oscillates during the light-dark cycle ${ }^{2}$, but it is difficult to work out where these genes fit into the clock mechanism because a clock can both measure and show time, those genes that are directly implicated in the clockwork need to be distinguished from those that mediate output signals.

In Neurospora, the endogenous clock is responsible for the precise circadian synchronization of cycles of asexual sporulation (conidiation), and the frequency ( $f r q$ ) gene seems to be a central component of the oscil- lator ${ }^{10}$. Transcription to produce frq messenger RNA is central to the clock, with levels of the frq transcript peaking in the morning. If an inducible frq gene is expressed at the wrong time, the conidiation rhythm is abolished; conversely, suppression of the inducible gene resets the conidiation cycle.

The frq messenger RNA transcript can be translated into protein from either of two initiation sequences (codons), resulting in the production of two Frq polypeptides ${ }^{11}$. Changes in temperature regulate the choice of codon used, setting the physiological temperature limits for rhythmicity. Immediately after synthesis, phosphate groups are added to both proteins (phosphorylation), yielding several forms of $\mathrm{Frq}^{12}$. It is not clear how Frq contributes to the generation of circadian rhythmicity, but it is probably at the level of transcription - indeed, Frq needs to be in the nucleus (where transcription occurs) to be active ${ }^{10}$. Moreover, although Frq does not belong to any well-defined class of transcription factor, it has several features that are typical of such factors: these include a putative helix-turn-helix domain through which it can bind DNA; a signal that allows it to be targeted to the nucleus (a nuclear-localization signal); and highly charged regions that

\title{
Molecular clocks in development
}

During the formation of

an embryo, the events

that lead to

determination of the

various cell types seem

to be controlled by

developmental timers

which operate within

individual cells. A

remarkable example of

this timekeeping is

provided by the transition

from rapid and

symmetrical cell

divisions to slow and

asymmetrical divisions in

embryos of the toad

Xenopus laevis. This

transition always occurs

at the twelfth cleavage

after fertilization, when

the embryo consists of

just over 2,000 cells

known as blastomeres.

The timing of the

transition is controlled by a clock that is intrinsic to each blastomere - timing does not depend on cell-cell interactions ${ }^{33}$. Another example comes from precursor cells that differentiate to form oligodendrocytes, a type

of non-neuronal cell found in the vertebrate nervous system. When placed individually in single wells, these precursors divide a number of times before differentiating. Two daughter cells from the same precursor undergo a synchronized number of divisions, and differentiate at the same time ${ }^{34}$.

Developmental clocks such as these are probably distinct from those that govern circadian rhythms, although the molecular mechanisms that control proliferation and differentiation programmes are obviously intermingled with cellular oscillatory functions. For example, expression of the avian equivalent of the Drosophila gene hairy in the chick is controlled by a molecular clock that is linked to the formation of somites. Hairy is expressed in cyclic pulses with a periodicity of 90 minutes - exactly the time that it takes to form one somite. Movement of the pulses does not depend on cell displacement or on propagation of an activating signal, and each cell seems to have its own functional clock $^{35}$.

Because of its intrinsic free-running property, the cell division cycle must also be considered as a molecular clock. Most eukaryotic cells in culture undergo mitosis (nuclear division) with a periodicity of roughly 24 hours. Is this just coincidence, or were cells sensitive to light-dark cycles millions of years ago? If they were, what we study today as the cell cycle could represent a vestigial circadian rhythm.

P.S.-C. could be involved in transcriptional activation (Table 1).

\section{Association of clock molecules}

In Drosophila, at least two genes are involved in clock function, and the dynamic association between their products ensures correct circadian rhythmicity. One of these, period (per), was the first clock gene ever to be isolated ${ }^{13}$. Nonsense mutations in per (which lead to an abnormally shortened Per polypeptide) result in the loss of rhythmic locomotor activity in Drosophila. Longlasting phase shifts in locomotor activity are also observed when pulses of heat are given to transgenic flies bearing a heat-inducible copy of per. Missense mutations, which cause single amino-acid substitutions in Per, change the length of the circadian cycle ${ }^{2,14}$.

Cyclical expression of per is regulated by transcription. The promoter region of the per gene, which is required for initiation of transcription, is sufficient to confer oscillating transcriptional regulation on another gene $^{14}$. But production of a stable messenger RNA transcript would lead to the accumulation of this per messenger RNA and, hence, only minor oscillations. The trick that the per gene uses to ensure a strict rhythm is to have a relatively short messenger RNA halflife $^{15}$. This is likely to be a very efficient regulatory mechanism, probably involving the rhythmic activation of factors involved in RNA processing. A mechanism of this kind exists in the dinoflagellate Gonyaulax polyedra, for example - circadian expression of a gene correlates with the cyclic binding of a protein to the $3^{\prime}$ end of the messenger RNA, regulating the stability of the transcript ${ }^{16}$.

The second fly clock gene is timeless (tim). Mutations in tim have dramatic consequences on per (Fig. 2): the oscillatory expression is lost; phosphorylation of the Per protein is disrupted; and timedependent nuclear transport is abolished. Conversely, cyclic expression of tim is altered in per mutants ${ }^{17}$. The Tim protein seems to regulate Per by interacting with it to form a Per-Tim dimer. Per contains a region called the PAS domain (Table 1), which is necessary for protein-protein interactions ${ }^{18}$, and is now thought to be the 'signature' of this class of clock molecule. PAS domains were named after the three proteins in which this structural motif was first identified: Drosophila Per, the mammalian Arnt, and Sim, which is the product of the fly single-minded gene. These domains have since been found in several transcription factors, where they are thought to confer target-gene specificity, and they are often coupled to a DNAbinding domain (a basic helix-loop-helix or bHLH domain). Drosophila Per does not contain a bHLH domain, however, suggesting that it may regulate transcription without binding DNA.

Surprisingly, Tim does not seem to con- 